\title{
LA ADMINISTRACIÓN TRUMP, EL ASCENSO DE LA REPÚBLICA POPULAR CHINA Y EL NUEVO «INTERNACIONALISMO INDEPENDIENTE»
}

\author{
David J. García Cantalapiedra*
}

SUMARIO: 1. PRIMACÍA Y ORDEN INTERNACIONAL LIBERAL MULTILATERAL: ¿EL FIN DE UNA ERA?-2. LA ADMINISTRACIÓN TRUMP Y EL NUEVO «INTERNACIONALISMO INDEPENDIENTE».-3. PERCEPCIONES SOBRE EL ASCENSO DE CHINA Y LA ADMINISTRACIÓN TRUMP: ENTRE PRIMACÍA Y OSB.-4. CONCLUSIONES.

\section{PRIMACÍA Y ORDEN INTERNACIONAL LIBERAL MULTILATERAL: ¿EL FIN DE UNA ERA?}

1. En algunos momentos de la Guerra Fría (durante la Administración Truman, durante la Administración Nixon, y parcialmente por Carter y Reagan en Oriente Medio), y sobre todo tras la caída del Muro de Berlín y la desaparición de la Unión Soviética (URSS), se empezaron a plantear en sectores políticos, militares y académicos norteamericanos la conveniencia de adoptar una posición estratégica global que redujera los compromisos de Estados Unidos, minimizando el riesgo de participación en conflictos militares, permitiendo una posición más flexible y que maximizara las ventajas relativas norteamericanas: esto es, en lugar de la postura de preponderancia o primacía (Preponderance/Primacy), una de equilibrio de poder externo Offshore Balancing (OSB) ${ }^{1}$. Esta estrategia es un concepto estratégico utilizado por autores dentro del Realismo estructural y ofensivo, donde una gran potencia elige otras potencias regionales para controlar el surgimiento de potenciales potencias hostiles. Sin embargo, en el «momento unipolar» ${ }^{2}$ man-

* Profesor Contratado Doctor (Acreditado a TU) en la Universidad Complutense de Madrid (djgarcia@pdi.ucm.es).

1 Por ejemplo, Layne, C. y Schwarz, B., «American Hegemony-Without an Enemy», Foreign Policy, 1993, núm. 92; LAYNE, C., «From Preponderance to Offshore Balancing: America's Future Grand Strategy», International Security, vol. 22, 1997, núm. 1, pp. 86-124. Actualmente véase: MEARSHEIMER, J. y WALT, S., "The Case for Offshore Balancing. A Superior U.S. Grand Strategy», Foreign Affairs, vol. 95, 2016, núm. 4, pp. 70-83.

2 Krauthammer, C., "The Unipolar Moment», Foreign Affairs, America and the World, vol. 70, 1990/91, núm. 1, pp. 23-33. 
tuvieron la planificación de defensa y la estrategia de seguridad nacional de Estados Unidos en la postura de Primacía ${ }^{3}$. La Contención y los proyectos previos de Gran Estrategia producidos en la primera Administración Bush y la Administración Clinton establecían una postura de Primacía (otros la llaman hegemonía liberal). Mantenían los caracteres de Primacía que también acompañaban a la Contención: un orden internacional abierto a los intereses y valores de Estados Unidos, que incluye la institucionalización y promoción de la democracia y el libre mercado, con la centralidad de Estados Unidos en el sistema; y evitar el ascenso de una potencia hegemónica regional o global. La misma rationale establecida para el enfrentamiento con la URSS se va a mantener después en el proyecto del plan-director de planificación de defensa (Defense Planning Guidance) DPG 1992-1999:

"Our first objective is to prevent the re-emergence of a new rival, either on the territory of the former Soviet Union or elsewhere that poses a threat on the order posed formerly by the Soviet Union. This [...] requires that we endeavor to prevent any hostile power from dominating a region whose resources would, under consolidated control, be sufficient to generate global power [...]. Our strategy must now refocus on precluding the emergence of any potential future global competitor».

2. A pesar de ello, se planteaba cada vez más claramente un cambio estratégico ya a finales de la Administración Clinton:

«Most of the world's other major powers have made it into a central theme of their foreign policy to attempt to build counterweights to American power. This is in fact one of the main trends in international politics today» ${ }^{4}$.

3. El 11-S y la Gran Recesión de 2008, sin embargo, iban a ofrecer alternativas extremas de esa postura de Primacía: la Administración Bush, con una reedición de la estrategia reaganiana de Peace through Strengh en su versión más wilsoniana; y la Administración Obama, con una aproximación neo-nixoniana de Pentapolaridad y New Detente ${ }^{5}$. Este debate ha vuelto a plantearse, no solo con la nueva Administración Trump, sino durante la campaña electoral presidencial en Estados Unidos en 2016, pero se ha obscurecido, aunque generalmente mezclado y confundido con la visión para el sistema internacional (Weltanschauung) de los candidatos presidenciales, Hillary Clinton y Donald Trump. En términos generales, la candidata demó-

\footnotetext{
3 Para un análisis en profundidad de esta visión de Primacía y los debates estratégicos subsecuentes, véase: García CANTAlaPIEDRA, D., EEUU y la construcción de un Nuevo Orden Mundial: la Administración Bush, las Relaciones Transatlánticas y la Seguridad Europea 1989-1992. Tesis Doctoral. Departamento de Estudios Internacionales. Facultad de CC. Políticas y de la Administración, UCM. 2001; también García Cantalapiedra, D., «"Peace through Primacy”: la Administración Bush, la política exterior de EEUU y las bases de una primacía imperial; geopolítica, recursos energéticos y guerra al terrorismo», UNISCI Papers, 2003, núm. 30.

4 Rodman, P., Uneasy Giant: the Challenges to American Predominance, Washington D. C., Nixon Center, 1999, p. 2.

5 García Cantalapiedra, D., «La Doctrina Obama, la teoría de la Guerra Limitada y la nueva política exterior de EEUU: ¿hacia una política neo-nixoniana?», UNISCI Discussion Papers, 2012, núm. 28, pp. 145-153.
} 
crata parecía defender un orden internacional liberal multilateral que hasta ahora defendía Estados Unidos a través de diferentes instrumentalizaciones de su postura de Primacía, aunque matizada por la visión neo-nixoniana de la Administración Obama. Por el contrario, el candidato republicano parecía optar por una postura sumamente crítica con el orden internacional liberal multilateral, y con una aproximación estratégica más en la línea del OSB, con ciertos toques nacionalistas (Alt-Right) y jacksonianos ${ }^{6}$.

\section{LA ADMINISTRACIÓN TRUMP Y EL NUEVO «INTERNACIONALISMO INDEPENDIENTE»}

4. Sin embargo, también hay posturas que establecen que los primeros pasos de la Administración Trump tienen afinidad con la visión del Internacionalismo Independiente ${ }^{7}$ de los años veinte, llevado a cabo por varios presidentes republicanos, en cuanto su visión nacionalista, maximizando su capacidad de maniobra y flexibilidad estratégica, y sus políticas proteccionistas y anti-inmigración ${ }^{8}$. No obstante, esas administraciones no eran tan aislacionistas como pudieran parecer (a pesar de la aparición del American First Committee) ya que trabajaban con otras grandes potencias (aunque sin un marco general global, ya que rechazaban la Sociedad de Naciones, las organizaciones internacionales y las llamadas entangling alliances) $)^{9}$ en el Pacto Briand-Kellog, las Conferencias de Ginebra, las Conferencias navales de Washington, o la política hacia China, en la creencia en que una economía en crecimiento y expansión reduciría el impacto que había tenido la Primera Guerra Mundial. Evidentemente, hay autores que advierten que el uso de una analogía histórica de este tipo puede ser problemática debido a que los factores y el marco internacional son diferentes en cada momento histórico ${ }^{10}$. Por ejemplo, a pesar de la retórica adoptada de retorno al Peace through Strengh reaganiano (National Security Presidential Memorandum 1) ${ }^{11}$, esto no va a suponer el ambicioso programa de rearme de aquel momento ${ }^{12}$. Se echa en

6 Russel Mead, W., «The Jacksonian Tradition and American foreign policy», The National Interest, Winter 1999/2000, pp. 9-29.

7 Hoff Wilson, J., American Business \& Foreign Policy 1920-1933, Lexington, U. Kentucky Press, 1971.

8 Buchanan, P., «It's Trump's Party, Now», Chronicles: A Magazine of American Culture, 3 de marzo de 2017, https://www.chroniclesmagazine.org/its-trumps-party-now/ (consultado el 1 de abril de 2017).

9 Sin embargo, hay estudios que parecen refutar esta vieja narrativa en la política exterior de Estados Unidos. Véase: Fromkin, D., «Entangling Alliances», Foreign Affairs, vol. 48, 1970, núm. 4, pp. 688700. Beckley, M., «The Myth of Entangling Alliances. Reassessing the Security Risks of U.S. Defense Pacts», International Security, vol. 39, 2015, núm. 4, pp. 7-48.

10 Foong Khong, Y., Analogies at War: Korea, Munich, Dien Bien Phu, and the Vietnam Decisions of 1965, Princeton, Princeton University Press, 1993.

11 «Section 1. Policy. To pursue Peace through Strength, it shall be the policy of the United States to rebuild the U.S. Armed Forces», The White House. Presidential Memorandum-Rebuilding US Armed Forces, 27 de febrero de 2017, https://www.whitehouse.gov/the-press-office/2017/01/27/presidential-memorandum-rebuilding-us-armed-forces (consultado el 1 de abril de 2017).

12 Se espera un incremento de "solo» 54.000 millones de dólares. OMB, Budget of the United States Government, Fiscal Year 2017, https://www.govinfo.gov/content/pkg/BUDGET-2017-BUD/pdf/BUDGET- 
falta una visión o plan comprehensivo oficial, y la tardanza en construir su administración han producido pocos documentos de significancia en seguridad nacional. A pesar de ello, hay en marcha un proceso de revisión de la estrategia de seguridad nacional y de la postura nuclear de Estados Unidos (Nuclear Posture Review) ${ }^{13}$, junto con un programa de modernización de las fuerzas nucleares norteamericanas en diez años que significará unos 400.000 millones de dólares ${ }^{14}$. No obstante, se puede atisbar ciertamente una aproximación diferente y un proceso de cambio: en este sentido, el Secretario de Estado Rex Tillerson, básicamente establece que la misión que tenía el Departamento de Estado desde 1945 hasta hoy va a cambiar ${ }^{15}$. Realmente (y aunque no de una manera totalmente ortodoxa desde el punto de vista de la escuela realista ${ }^{16}$, se había iniciado un proceso de abandono de la postura de Primacía durante la Administración Obama, con la adopción de una visión de tipo multipolar (según la Estrategia de Seguridad Nacional de 2010) y con una prioridad en el fortalecimiento de alianzas, tal como la política Pivot to Asia a partir de 2011. Esta visión ha sido muy criticada también desde sectores que defienden la posición de Primacía (Robert Lieber, Eliot Cohen, Kori Shake) ${ }^{17}$, Josep Joffe ${ }^{18}$, o de la persistencia de la «Unipolaridad» como Stephen Brooks y William C. Wohlforth ${ }^{19}$ entre otros.

2017-BUD.pdf (consultado el 20 de abril de 2017); https://www.nytimes.com/2017/02/27/us/politics/ trump-budget-military.html?_r=O (consultado el 20 de abril de 2017).

${ }_{13}$ Véase la entrevista de Christopher Ford, senior director for weapons of mass destruction and Counterproliferation del NSC, Carnegie Nuclear Policy Conference 2017, 21 de marzo de 2017, http://carnegieendowment.org/2017/03/21/morning-keynote-with-nsc-senior-director-christopher-ford-pub-68162 (consultado el 1 de abril de 2017). Los cambios con respecto al Nuclear Posture Review de 2010 parece que van a ser tan profundos que Ford no proporcionó información clara y precisa sobre varios temas en cuanto a desarme y la política de empleo sobre armas nucleares (Nuclear Weapons Employment Policy) aunque sí se atisba un cambio de posición en la Administración Trump respecto a la de la Administración Obama.

${ }_{14}$ Congressional Budget Office, Projected Costs of U.S. Nuclear Forces, 2017 to 2026, febrero de 2017, https://www.cbo.gov/sites/default/files/115th-congress-2017-2018/reports/52401-nuclearcosts.pdf (consultado el 21 de abril de 2017).

${ }_{15}$ Tillerson, R. W., Secretary of State, Remarks to U.S. Department of State Employees, Dean Acheson Auditorium Washington, D. C., 3 de mayo de 2017, https://www.state.gov/secretary/remarks/2017/05/270620.htm (consultado el 8 de mayo de 2017).

16 Walt, S., «Obama Was Not a Realist President», Foreign Policy, 7 de abril de 2016, http:/fforeignpolicy.com/2016/04/07/obama-was-not-a-realist-president-jeffrey-goldberg-atlantic-obama-doctrinel (consultado el 10 de enero de 2017).

${ }_{17}$ SchaKe, K., «The False Logic of Retreat: Will Washington abandon the Order?», Foreign Affairs, vol. 96, 2017, núm. 1, pp. 41-46; LIEBER, R., Retreat and Its Consequences: American Foreign Policy and the Problem of World Order, Cambridge, Cambridge University Press, 2016; CoHen, E., The Big Stick: The Limits of Soft Power and the Necessity of Military Force, Nueva York, Basic Books, 2016.

18 Joffe, J., «Obama Is Not a Realist. He's an isolationist with drones and special-operations forces», The Atlantic, 10 de marzo de 2016, https://www.theatlantic.com/international/archive/2016/03/ obama-doctrine-goldberg-realist-isolationist/473205/ (consultado el 5 de febrero de 2017); SANDERS, P., "Obama is not a Realist», The National Interest, 26 de agosto de 2014, http://nationalinterest.org/feature/ barack-obama-not-realist-11124 (consultado el 5 de febrero de 2017).

19 Brooks, S. y Wohlforth, W., «The Rise and Fall of the Great Powers in the Twenty-first Century: China's Rise and the Fate of America's Global Position», International Security, vol. 40, 2015/16, núm. 3, pp. 7-53; id., World Out of Balance: International Relations and the Challenge of American Primacy, Princeton, Princeton University Press, 2008; id., "Assessing the Balance», Cambridge Review of In- 


\section{PERCEPCIONES SOBRE EL ASCENSO DE CHINA Y LA ADMINISTRACIÓN TRUMP: ENTRE PRIMACÍA Y OSB}

5. Sin embargo, la cuestión más importante en este debate es probablemente si Estados Unidos va a abandonar los dos postulados básicos de su postura estratégica que se remontan a la Segunda Guerra Mundial: el mantenimiento de un orden internacional favorable a los intereses y valores de Estados Unidos, y evitar el ascenso de una potencia regional que dominando una región clave consiguiera los recursos suficientes para convertirse en un aspirante global ${ }^{20}$. En este sentido, el ascenso de la República Popular China (RPC) es central en este debate, tanto desde el punto de vista material como perceptivo y narrativo ${ }^{21}$. Incluso en este último aspecto el llamado «ascenso pacífico» de China ha sido una construcción narrativa occidental, interpretando los discursos del Primer Ministro Wen Jibao en 2003, cuando ni siquiera era una postura oficial adoptada por el propio gobierno chino, que se enfocaba más en el concepto "paz y desarrollo» ${ }^{22}$. Por otro lado, autores como Graham Allison aventuran la Trampa de Tucídides ${ }^{23}$ para argumentar el rumbo de colisión de Estados Unidos y la RPC, en la línea de los realistas ofensivos y algunos defensivos ${ }^{24}$. Mientras, las posiciones liberales razonan contrariamente debido a los intereses comunes, las instituciones internacionales y las limitaciones internas, sobre todo debido a que el crecimiento y expansión económica de la RPC está haciendo el país más abierto y dependiente de la economía global ${ }^{25}$. Sin embargo, la conducta de la RPC muestra

ternational Affairs, vol. 20, 2011, núm. 2, pp. 201-219; LIEBER, R., Power and Willpower in the American Future: Why the United States Is Not Destined to Decline, Cambridge, Cambridge University Press, 2012.

20 García Cantalapiedra, D., op. cit., nota 3.

21 Foong Khong, Y., «Primacy or World Order? The United States and China's Rise. A Review Essay», International Security, vol. 38, 2013/14, núm. 3, pp. 153-175; Schweller, R. y Pu, X., "After Unipolarity: China's Visions of International Order in an Era of U.S. Decline», International Security, vol. 36, 2011, núm. 1, pp. 41-72.

22 Véase en este particular García Cantalapiedra, D., «La Política exterior de EEUU hacia China y la dinámica de seguridad en Asia: impactos regional y global», Cursos de Derecho Internacional Público y RR.II. de Victoria-Gasteiz, 2015, Cizur Menor, Aranzadi, 2016, pp. 285-287.

${ }^{23}$ Allison, G., «Destined for War: Can America and China Escape Thucydides's Trap?», http:// www.belfercenter.org/thucydides-trap/book/thucydides-author; $i d$., "The Thucydides Trap: Are the U.S. and China Headed for War?», The Atlantic, 24 de septiembre de 2015, https://www.theatlantic.com/international/archive/2015/09/united-states-china-war-thucydides-trap/406756/ (consultado el 5 de febrero de 2017).

24 Mearsheimer, J., «China's Unpeaceful Rise», Current History, vol. 105, 2006, núm. 690, pp. 16063; también en este sentido: SIDDAL, A., "The Misapplication of Defensive Realism: the Security Dilemma and Rising Powers in East Asia», The 42nd APSA Annual Conference, 3-6 de octubre de 2000; Mearsheimer, J., "Can China Rise Peacefully?», The National Interest, 25 de octubre de 2014, http:// nationalinterest.org/commentary/can-china-rise-peacefully-10204 (consultado el 5 de febrero de 2017); Christensen, T., «The Advantages of an Assertive China: Responding to Beijing's Abrasive Diplomacy», Foreign Affairs, vol. 90, 2011, núm. 2, pp. 54-67; SwaIne, M. y FrafEL, M. T., "China's Assertive Behavior-Part Two: The Maritime Periphery», China Leadership Monitor, 35, 2011a, pp. 2-29, https:// taylorfravel.com/documents/research/fravel.2011.CLM.maritime.periphery.pdf (consultado el 5 de febrero de 2017); Liff, A. e IKenberry, G. J., «Racing toward Tragedy? China's Rise, Military Competition in the Asia Pacific, and the Security Dilemma», International Security, vol. 39, 2014, núm. 2, pp. 52-91.

${ }_{25}$ Zoellick, R., "Whither China: from Membership to Responsibility», National Committee on USChina Relations, 21 de septiembre de 2005, https://2001-2009.state.gov/s/d/former/zoellick/rem/53682. 
una mayor agresividad hacia sus vecinos en diferentes disputas territoriales, independientemente de si son o no aliados de Estados Unidos, cuestión que respalda la postura de los realistas ofensivos que establecen la búsqueda de la hegemonía por parte de la RPC en la zona. Así, la ruptura de las reglas de conducta y del orden internacional liberal multilateral (el statu quo en el Mar del Sur de China o las islas Senkaku y la Convención sobre el Derecho del Mar de 1982 ${ }^{26}$ se podría ver como un incentivo para la Administración Trump para aplicar su internacionalismo independiente, por ejemplo, tal como mostró en cuanto a Taiwan y la política de Una Sola China (One China policy) o en el caso de Corea del Norte ${ }^{27}$. Parece, además, que se está produciendo cierta competición militar causada por dilemas de seguridad que, aunque aún no señalan una carrera de armamentos generalizada, no obstante muestran una dinámica gradual entre China y otros Estados hacia progresivas y cada vez mayores inversiones en capacidades militares que pueden empeorar cada más en el futuro ${ }^{28}$. Sin embargo, también parece que existen dilemas de seguridad producidos por conflictos de intereses que se ven por los actores como búsquedas de alteración del statu quo que no responden a búsquedas de seguridad ${ }^{29}$.

\section{CONCLUSIONES}

6. En este sentido, Estados Unidos y la RPC se pueden encontrar en una situación en la que la incertidumbre, los errores de percepción y la falta de

htm (consultado el 5 de febrero de 2017); Callahan, B., «John Mearsheimer and Joseph S. Nye Jr. on the rise of China and America's engagement policy», The Diplomat, 8 de julio de 2015; JoHnson, A., "Is China a Status Quo Power?», International Security, vol. 27, 2003, núm. 4, pp. 5-56; NYE, J., "China's Re-Emergence and the Future of the Asia-Pacific», Survival, vol. 39, 1997, núm. 4, pp. 65-79.

${ }^{26}$ La Corte Permanente de Arbitraje resolvió el 12 de julio de 2016 la petición de la República de Filipinas contra la República Popular de China en relación a las controversias entre ambos Estados sobre los derechos marítimos y prestaciones en el mar del Sur de China, el estado de ciertas características geográficas en la zona, así como la legalidad de diversas acciones por parte de Pekín en esas aguas, de acuerdo a la Convención sobre Derecho del Mar de Naciones Unidas de 1982. La Corte falló que no había base legal para la reivindicación por parte de China de derechos históricos sobre los recursos dentro de las áreas marinas que entran en la llamada «Nine-Dash Line».

27 Aunque posteriormente el propio Presidente Trump reafirmaría esta política. Sobre ella véase: Congressional Research Service, China/Taiwan: Evolution of the "One China» Policy-Key Statements from Washington, Beijing, and Taipei, 10 de octubre de 2014, https://fas.org/sgp/crs/row/RL30341.pdf (consultado el 5 de febrero de 2017). Sin embargo, "The United States did not explicitly state Taiwan's status in the U.S.-PRC Joint Communiques of 1972, 1979, and 1982. The United States "acknowledged" the "one China" position of both sides of the Taiwan Strait»; sobre Corea del Norte véase U. S. Department of Defense, Joint Statement by Secretary of State Rex Tillerson, Secretary of Defense James Mattis, Director of National Intelligence Dan Coats, Press Operations, 26 de abril de 2017, https://www.defense. gov/News/News-Releases/News-Release-View/Article/1163940/joint-statement-by-secretary-of-state-rextillerson-secretary-of-defense-james/ (consultado el 27 de abril de 2017).

28 LIFF, A. e IKENBERRY, G. J., op. cit., nota 24.

29 Jervis, R., "Cooperation under the Security Dilemma», World Politics, vol. 30, 1978, núm. 2, pp. 167-214; Glaser, C., «The Security Dilemma revisited», World Politics, vol. 50, 1997, núm. 1, pp. 171-201. Para una revisión crítica de ambos, véase TANG, S., «The Security Dilemma: A Conceptual Analysis», Security Studies, vol. 18, 2009, núm. 3, pp. 587-623. 
confianza estratégica amplifiquen las opciones de conflicto más allá de la dinámica del dilema de seguridad, en una interpretación de la ruptura del statu quo en Asia-Pacífico. El ritmo y escala del aumento de la capacidad militar de Beijing, su tendencia a rechazar las preocupaciones de otros Estados y su poca transparencia acerca de sus gastos militares, capacidades e intenciones parece que están exacerbando la percepción de amenaza en la conducta de la RPC. Esta tendencia podría representar un argumento en las posiciones que defienden el ascenso de la RPC como una amenaza al statu quo, no solo de la zona, sino del orden internacional. En este sentido, la Administración Trump al elegir una OSB, más en la línea de su Internacionalismo Independiente, dependería de sus aliados en la zona, los cuales no tienen la capacidad por sí solos de reequilibrar a la RPC. Paradójicamente Estados Unidos se podría encontrar en un conflicto militar en esta situación más fácilmente, o que su actitud es interpretada como un buckpassing por su aliados, y estos reconsideren su política hacia Estados Unidos y la RPC. Sin embargo, dentro del contexto de la dinámica de seguridad en Asia-Pacífico, y la multiplicación de problemas y opciones estratégicas en la zona, esto es, Corea del Norte, el Mar del Sur de China, las islas Senkaku y las relaciones India-RPC, se proyecta un escenario que aumenta peligrosamente las opciones de conflicto en el futuro, ya que produce una escalada gradual de tensión que podría derivar en un enfrentamiento militar no deseado, con consecuencias de difícil previsión.

Palabras clave: Estados Unidos, China, Trump, Asia.

Keywords: United States, China, Trump, Asia. 